\title{
Highly variable sperm precedence in the stalk-eyed fly, Teleopsis dalmanni

\author{
Laura S Corley ${ }^{1,2}$, Samuel Cotton ${ }^{1}$, Ellen McConnell ${ }^{1}$, Tracey Chapman ${ }^{3}$, \\ Kevin Fowler ${ }^{1}$ and Andrew Pomiankowski*1
}

Address: ${ }^{1}$ Galton Laboratory, Department of Biology, University College London, Wolfson House, 4 Stephenson Way, London NW1 2HE, UK, ${ }^{2}$ Department of Entomology, Washington State University, Pullman WA 99164-6382, USA and ${ }^{3}$ Department of Biology, University College London, Darwin Building, Gower Street, London, WC1E 6BT, UK

Email: Laura S Corley - corley@mail.wsu.edu; Samuel Cotton - s.cotton@ucl.ac.uk; Ellen McConnell - e.mcconnell@ucl.ac.uk; Tracey Chapman - t.chapman@ucl.ac.uk; Kevin Fowler - k.fowler@ucl.ac.uk; Andrew Pomiankowski* - ucbhpom@ucl.ac.uk

* Corresponding author

Published: 26 June 2006

BMC Evolutionary Biology 2006, 6:53 doi:10.1/86/147|-2|48-6-53

This article is available from: http://www.biomedcentral.com/I47I-2/48/6/53

(c) 2006 Corley et al; licensee BioMed Central Ltd.

This is an Open Access article distributed under the terms of the Creative Commons Attribution License (http://creativecommons.org/licenses/by/2.0), which permits unrestricted use, distribution, and reproduction in any medium, provided the original work is properly cited.
Received: 10 March 2006

Accepted: 26 June 2006

\begin{abstract}
Background: When females mate with different males, competition for fertilizations occurs after insemination. Such sperm competition is usually summarized at the level of the population or species by the parameter, $P_{2}$, defined as the proportion of offspring sired by the second male in double mating trials. However, considerable variation in $P_{2}$ may occur within populations, and such variation limits the utility of population-wide or species $P_{2}$ estimates as descriptors of sperm usage. To fully understand the causes and consequences of sperm competition requires estimates of not only mean $P_{2}$, but also intra-specific variation in $P_{2}$. Here we investigate within-population quantitative variation in $P_{2}$ using a controlled mating experiment and microsatellite profiling of progeny in the multiply mating stalk-eyed fly, Teleopsis dalmanni.

Results: We genotyped $38 \mathrm{I}$ offspring from 22 dam-sire pair families at four microsatellite loci. The mean population-wide $P_{2}$ value of 0.40 was not significantly different from that expected under random sperm mixing (i.e. $P_{2}=0.5$ ). However, patterns of paternity were highly variable between individual families; almost half of families displayed extreme second male biases resulting in zero or complete paternity, whereas only about one third of families had $P_{2}$ values of 0.5 , the remainder had significant, but moderate, paternity skew.

Conclusion: Our data suggest that all modes of ejaculate competition, from extreme sperm precedence to complete sperm mixing, occur in $T$. dalmanni. Thus the population mean $P_{2}$ value does not reflect the high underlying variance in familial $P_{2}$. We discuss some of the potential causes and consequences of post-copulatory sexual selection in this important model species.
\end{abstract}

\section{Background}

When females copulate with more than one partner, competition for fertilizations occurs after insemination. Such post-copulatory sexual selection can be a potent evolutionary force, as is evidenced by the numerous male behavioral, physiological, and morphological adaptations that influence sperm competition, such as mate guarding, increased copulation duration, seminal fluid induced reluctance of female re-mating, and the mechanical removal of sperm [1-3]. In addition, post-copulatory 
sexual selection can enhance or diminish male ornament evolution if ornament size covaries positively or negatively, respectively, with sperm competitive ability [4-6].

The most widely used metric for sperm competition that is used to infer patterns of paternity is the proportion of eggs sired by the second male in controlled double-mating trials $\left(P_{2} ;[7]\right)$. Species or population level studies of $P_{2}$ have been used extensively to describe sperm competition, particularly in insects [2] and birds [1]. However, considerable variation in $P_{2}$ often occurs between populations and individuals, and intra-specific values of $P_{2}$ can range from zero to one $[2,5,8]$. Such variation can severely limit the utility of population-wide (or species) $P_{2}$ estimates as descriptors of sperm usage, because it fails to account for variation in male performance $\left(P_{2}\right.$ is derived from the performance of both first and second males), or aspects of female morphology and behaviour, such as sperm storage, that may differ between individual females [9]. For example, within many Lepidopteran species, some females lay eggs fertilized almost exclusively by the first male to mate, whereas others show strong second male sperm precedence ([10]; see also [11] for an example in guppies); in these instances mean $P_{2}$ values do not reflect the underlying bimodal distribution of male fertilization success. So in order to fully understand the causes and consequences of sperm competition it is necessary to estimate not only mean levels of sperm precedence, but also intra-specific variation around that mean.

Numerous factors have been shown to influence intraspecific variation in $P_{2}$ including male size, sexual ornamentation, mating history, reproductive organ size and diet $[2,3,5]$. There is also support for the hypothesis that males tailor their ejaculate in response to female factors such as size, mating status, age, fecundity and familiarity [12]. In addition, evidence is accumulating that, contrary to traditional views, ejaculates are in fact costly and cannot be produced in limitless quantities $[12,13]$, and that male gamete availability can limit zygote formation. Under sperm-limitation, reproductive asymmetry between the sexes for variance in fertilisation success, and with it the advantage of sperm competition for available ova, will be reduced. Nonetheless, if a particular mating role is favoured (e.g. first rather than last), a male is still predicted to invest more in the favoured role [14]. Spermlimitation phenotypes are most commonly seen in freespawning external fertilizers at low density [15-17], although similar selective environments may be common in internal fertilisers when females receive insufficient sperm to fertilise all of their eggs [e.g. [12,18]].

Stalk-eyed flies (Diptera:Diopsidae) are increasingly important model organisms for studies of sexual selection [19-22]. They are characterised by the lateral extension of the eyes on elongate protuberances on the side of the head capsule, a trait common to both sexes in all species $[19,23]$. In many species the eyespan of males is greater than that of females, the result of sexual selection through female mate choice [24-29] and male-male competition $[30,31]$.

The Malaysian stalk-eyed fly, Teleopsis dalmanni (formerly known as Cyrtodiopsis dalmanni; [32]), exhibits extreme sexual dimorphism for eyespan resulting from strong intra- and inter-sexual selection on the trait in males (ibid.). Females form large harems on root hairs overhanging the eroded banks of streams and males compete to control these harems $[19,33]$. Both sexes are highly promiscuous and mate at high frequencies ( 10 and 6 times per hour in the laboratory, for males and females respectively; $[34,35])$. There is also some evidence that males strategically allocate more ejaculate to larger, more productive females through the production and delivery of larger spermatophores [36]. However, T. dalmanni spermatophores are small [37] and females store few sperm following a single mating ( 140; [36]). Females are therefore sperm-limited and must copulate repeatedly to attain maximal fertility [38]. This problem is exacerbated in large, highly fecund females despite being allocated more ejaculate, as they lay a lower proportion of fertilised eggs following a single copulation in comparison with less productive females [38]. Without measures of paternity however, the value of both mate choice and multiple mating can only be inferred indirectly.

Previous research describing patterns of paternity in a congener, T. whitei, provides ambiguous evidence about patterns of sperm use $[39,40]$. Using an irradiated sterile male technique, Lorch et al. [39] reported that T. whitei exhibits first male sperm precedence. Irradiated (I) and non-irradiated $(\mathrm{N})$ males were mated sequentially to females in a reciprocal mating design, and evidence for first male precedence was inferred as NI families produced offspring more frequently than IN families. Lorch et al. [39] also concluded that sperm mixing was important since some IN crosses produced pupae. In another study [40], female T. whitei were mated to a male from two different populations 24 hours apart, and offspring paternity was assigned using heritable inter-population differences in leg colour. Wilkinson and Fry [40] found no significant effect of mating order on patterns of paternity with both first and second males siring equal numbers of progeny, suggesting that sperm mixing $\left(P_{2} \approx 0.5\right)$ is the predominant mode of sperm utilization. Wilkinson and Fry [40] also found some evidence for intra-specific variation in $P_{2}$, as males carrying a meiotic drive chromosome produced fewer sperm than non-drive males, and hence suffered a reduction in their proportion of progeny sired. 
As yet, there has been no effort to identify within-population quantitative variation in $P_{2}$ in stalk-eyed flies. In this paper we investigated intra-specific, within population variation of $P_{2}$ in $T$. dalmanni using controlled mating experiments and microsatellite profiling of progeny. We standardized male mating history, diet and body size in order to limit variation in male reproductive organ size, as we have shown that testes and accessory glands are reduced by mating [41] and scale positively with diet quality and body size [Rogers et al. in prep; Cotton \& Pomiankowski unpublished]. We also chose sires with similar eyespan to each other to limit the potential effects on $P_{2}$ of female mate choice based on male ornament size $[25,27,28]$. We show that even in the absence of these factors there is significant variation in sperm precedence in $T$. dalmanni, with familial $P_{2}$ values ranging from zero to one. We discuss the likely causes of this variation.

\section{Results}

A total of 662 progeny were collected from the 22 damsire pair families (mean total brood size \pm S.D. $=30.09 \pm$ 16.09 , range 8 - 63), of which 381 were genotyped successfully at four microsatellite loci (mean genotyped brood size \pm S.D. $=17.32 \pm 7.92$, range $5-35$ ). The mean value ( \pm S.D.) of $P_{2}$ across families was $0.40 \pm 0.38$. This average is not significantly different from a $P_{2}$ of $0.5(t=$ 1.16 , d.f. $=21, P=0.26$ ).

We found significant variation between the different families in terms of their $P_{2}\left(G_{H}=292.09\right.$, d.f. $\left.=21, P<0.001\right)$. The pattern is approximately tri-modal, with $P_{2}$ peaks at approximately 0, 0.5 and 1 (Figure 1). Ten families (45\%) exhibited extreme second male biases resulting in zero or complete paternity ( $n=1$ and 3, respectively; Fig. 1). These results were unlikely to be due entirely to one of the males being infertile in all three of their matings, as the incidence of $P_{2}=0$ or 1 was greater than that expected given the level of male infertility (over three matings) in the population $\left(22.7 \%\right.$ vs. $12.5 \%$, see Methods $\S \chi^{2}=$ 9.14, d.f. $=1, P=0.002$ ). Moreover, heterogeneity of $P_{2}$ was not entirely attributable to these families, as a replicated goodness-of-fit test using families with $P_{2} \neq 0$ or 1 was still significant $\left(G_{H}=60.55\right.$, d.f. $\left.=11, P<0.001\right)$. Eight families (36\%) displayed patterns of random paternity (i.e. their $P_{2}$ was not significantly different from 0.5 ; Figure 1). The remainder had significant moderate (but not extreme) paternity skew, reflecting both first and second male sperm precedence (Figure 1).

First and second males did not differ consistently from each other for eyespan $(t=1.21$, d.f. $=21, P=0.24)$ or thorax length $(t=0.00$, d.f. $=21, P=1.00)$. Male morphology did not explain variation in $P_{2}$ (first male eyespan $r=$ 0.28 , d.f. $=21, P=0.20$, second male eyespan $r=-0.16$, d.f. $=21, P=0.47$, first male thorax $r=0.10$, d.f. $=21, P=$

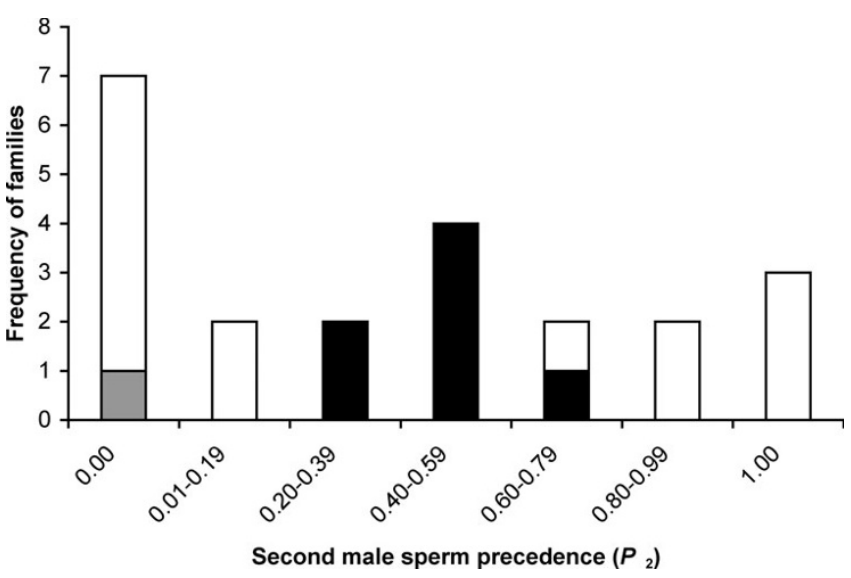

Figure I

The distribution of second male sperm precedence $\left(P_{2}\right)$ among 22 dam-sire pair families in the stalk-eyed fly, Teleopsis dalmanni. Open bars depict the frequency of families with $P_{2}$ significantly different from 0.5 (using 2-tailed binomial tests); black bars denote those with $P_{2}$ not significantly different from 0.5. The grey bar denotes the sperm precedence of a single family where the observed $P_{2}$ of zero was not significantly different from $P_{2}=0.5$. However, the brood size of this family was small $(n=5)$, so this result should be treated with caution.

0.65 , second male thorax $r=-0.11$, d.f. $=21, P=0.62)$. Relative male eyespan did not explain variation in $P_{2}$ (thorax included in model: first male relative eyespan $F=2.91$, d.f. $1,19, P=0.10$, second male relative eyespan $F=0.27$, d.f. $=1,19, P=0.61$ ), and neither did the difference in morphology between first and second males explain variation in $P_{2}$ (eyespan difference $r=-0.09$, d.f. $=21, P=0.68$, thorax difference $r=0.16$, d.f. $=21, P=0.47$ ). Female body size (thorax length) had no significant effect on $P_{2}(r$ $=-0.10$, d.f. $=19, P=0.66$ ), and neither did female eye$\operatorname{span}(r=-0.37$, d.f. $=19, P=0.10)$, although the latter did reveal a trend for females with larger eyespans to produce broods with fewer offspring sired by the second male.

Our data are unlikely to be affected by biased sampling of progeny within broods (with respect to male order) or variable brood sizes, as there was no correlation between the proportion of the brood sampled and $P_{2}(r=0.15$, d.f. $=21, P=0.51)$, or between total or genotyped brood size and $P_{2}$ (total brood size $r=-0.03$, d.f. $=21, P=0.89$; genotyped brood size $r=0.03$, d.f. $=21, P=0.91$ ).

\section{Discussion}

Using controlled mating experiments, assigning paternity using microsatellite markers, we demonstrated that sperm usage is highly variable in the stalk-eyed fly $T$. dalmanni (Figure 1). Almost half of the dam-sire pair families genotyped had extreme first or second male paternity biases 
( $P_{2}=0$ or 1 , respectively). Sperm precedence was not the only mode of sperm utilization, since the null model of random sperm mixing (i.e. $P_{2}=0.5$ ) explained patterns of paternity in over one third of families. Furthermore, a number of families displayed first or second male sperm precedence in conjunction with varying degrees of sperm mixing, resulting in moderate, but significant, paternity biases (i.e. $0<P_{2}<0.5$ and $0.5<P_{2}<1$, respectively). Thus all modes of sperm usage were found in T. dalmanni, and $P_{2}$ exhibited a tri-modal distribution (Figure 1).

What are the causes of such variation in paternity? We can exclude some factors which our experimental design deliberately set out to minimise. It is unlikely that our data can be explained by (cryptic) female choice for male ornaments or other aspects of external morphology, a phenomenon seen in numerous other species $[1-3,5]$, since first and second males did not differ in eyespan, the male sexual trait, or body size. As male eyespan is an accurate indicator of accessory gland and testis size in $T$. dalmanni [Rogers et al. in prep; Cotton \& Pomiankowski unpublished], differences in reproductive organ size are also unlikely to be explanatory variables, even though we did not explicitly measure these traits.

Male mating history is often associated with changes in male investment in current mating attempts, with concomitant effects on patterns of paternity $[12,13]$. However, all males were virgins at the start of the experiment and performed equal numbers of copulations, so variation in mating experience did not differ between first and second males, and hence is not an explanation of our data. In addition, all males were maintained on a high quality diet, so variation in environmental conditions was minimised between pairs of sires. These factors (when variable) may well be important in determining stalk-eyed fly paternity and deserve further investigation, but they cannot explain the results reported in the current study.

In contrast, female mating history was not constant across sires (only the first male mated with a virgin). In promiscuous species, such as T. dalmanni, it is likely to be advantageous for a male to mate with a virgin female as they only risk defence, not offence, sperm competition, and theory suggests that males should on average invest more in their favoured mating role (i.e. first vs. second) when females are sperm limited [14]. However, this hypothesis can be refuted, as the average value of $P_{2}$ was not significantly different from 0.5 .

Another possible explanation is that male infertility explains the variation in $P_{2}$ seen in our study. A recent simulation study showed that tri-modal distributions can be generated by male infertility in the range $10-30 \%$, given that relative fertilization success is randomly allocated to each male [18]. Whilst this simulation study is not directly applicable to our experiment, it does point out that male infertility alone can generate a high frequency of $P_{2}=0$ or 1. Even though infertility is quite common in T. dalmanni (estimated as $12.5 \%$ for males mated three times [38]), it is not high enough to explain the incidence of families with extreme paternity bias in our study (almost half had $P_{2}=0$ or 1 , which would require a rate of infertility equal to $22.7 \%$ ). However, infertility seems a good explanation of a major part of the frequency of $P_{2}=0$ or 1 . In addition, partial infertility due to insemination failure of one or two of the three matings made by each male could account for a large fraction of the variation observed between $0<P_{2}<$ 1 . Another hypothesis that can generate a tri-modal distribution is "sloppy" sperm mixing. Harvey and Parker [42] report that when sperm from each male's ejaculate tend to clump together and females only use a small proportion of the sperm they receive to fertilise their eggs, high variance and multi-modal $P_{2}$ distributions will be common. There is no information about sperm usage in stalk-eyed flies, so it is difficult to evaluate this idea. Further experimental work is needed to evaluate the relative importance of these hypotheses in explaining our data.

It is possible that males varied their ejaculate expenditure in relation to the reproductive value of females, in conjunction with their perceived mating status, as is observed frequently in other species [reviewed in [12]]. Since large eyespan females have higher fecundity ([36]; Cotton \& Pomiankowski unpublished) and are also more sperm limited [36], first males are predicted to invest relatively more in large eyespan, virgin females. We found some support for this in the form of a (albeit non-significant) negative correlation between female eyespan and the $P_{2}$ of a brood; males mating first with a large eyespan female showed a tendency to sire more offspring that the subsequent male. Another possibility is that males varied in aspects of external morphology or behaviour, independent of male body and ornament size, which affected male fertility. For example, males may have differed in the size or shape of their intromittant organs [43] and/or their copulatory courtship behaviour and this may have altered their ability in sperm competition, as has been reported in other insects $[3,44,45]$.

The presence of such extreme variation in paternity means that post-copulatory sexual selection will have a profound influence on the effects of pre-mating biases, and may partially explain the high mating rate seen in $T$. dalmanni. Female T. dalmanni mate frequently with both the same and different males, and prefer to mate most often with large eyespan males $[25,27,28]$. Repeated mating is beneficial for females since it helps to alleviate sperm limitation and increases the number of fertile eggs $[36,38]$. A high mating rate is also beneficial for males as it increases 
the number of females inseminated in a harem, and also the sperm load within multiply mated individual females. In the context of the current study, the latter may be particularly important given inter-male competition and the high incidence of sperm mixing.

Variable $P_{2}$ means that pre-copulatory mate choice is somewhat paradoxical both in terms of direct fertility benefits and indirect "good genes" benefits. Superficially it appears that the male that a female prefers to mate with is not necessarily the male that will sire her offspring. This paradox can be resolved however, with the observation that preferred males with large ornaments also have larger testes and accessory glands [Rogers et al. in prep.; Cotton \& Pomiankowski unpublished]. Accessory gland size covaries both phenotypically [41,46] and genetically [47] with male mating frequency, and number of sperm stored in a female's spermathecae correlates positively with the testis size of her mate [48]. Therefore, the advantages of choice (both direct and indirect; Rogers et al. in prep; [49]) for exaggerated ornaments might be maintained because large eyespan males can minimise the uncertainty arising from variable paternity by mating more frequently and out numbering the sperm of other males. However, further experiments are required to specifically test these hypotheses.

\section{Conclusion}

We have shown that the pattern of sperm usage is highly variable in the stalk-eyed fly, T. dalmanni. This greatly limits the utility of population-based estimates of $P_{2}$ as descriptors of sperm usage, and suggests that sperm precedence should be viewed as context-specific, rather than a general, constant, metric in stalk-eyed flies. The unexplained variance in male fertilization success found by this study requires further investigation in order to evaluate potential causes and consequences.

\section{Methods}

\section{Study animal}

The laboratory population of $T$. dalmanni used in this experiment was derived from wild caught flies collected from Malaysia in 1993 by AP. Flies have been maintained in cage culture at $25^{\circ} \mathrm{C}$ on a 12:12 light/dark cycle, at high population size ( $>200$ individuals) to minimize inbreeding. This population does not carry $X$-linked meiotic drive, which is found in natural populations of $T$. dalmanni [50]. The light regime included a 15-min 'dawn' period in which the culture room was illuminated by a single $60 \mathrm{~W}$ light bulb. All observations of mating behaviour commenced at the start of the dawn period. Experimental flies were collected as eggs and reared under low larval density [51]. Emerging adults were segregated by sex and measured for eyespan and thorax length [51] to an accuracy of $0.01 \mathrm{~mm}$ using a monocular microscope and the image analysis program NIH Image (Version 1.55; National Institute of Health, Bethesda, MD, USA). Flies were measured "blind" by a single person (EM). All individuals used in this experiment were sexually mature (68 weeks post eclosion) at the start of each experiment and fed high quality food (puréed corn) ad libitum.

\section{Mating design}

Individual males were anaesthetized on ice and their left middle tibia was removed 2 weeks prior to the mating trials, to yield DNA for pre-screening microsatellite genotype $(n=96)$. Pilot mating trials showed that tibia removal had no effect on the ability of males to mount and mate with females. Males were genotyped at each of four microsatellite loci ([52], see below for details), and pairs were formed that maximised allelic differences between them, to give a total of $n=30$ pairs. All of these male pairs differed by at least one allele for at least two loci.

In the mating trials, a single virgin female was placed in a container with one randomly chosen male (male 1) from a pair of males, at artificial dawn (lights on). The female and male were observed until three matings of 30 seconds or longer had occurred (spermatophore transfer usually occurs after copulations of 30 seconds). The male was then removed. At dawn on the second day, the second male (male 2) from the pair was placed with the female, and observed until three matings of 30 seconds or longer had occurred. The second male was then removed. Three matings per male were used to reduce the incidence of complete infertility [38].

Mated females were provided with food and water ad libitum and eggs were collected every two days for the following 10 days. All females were frozen on the last day and their abdomens were used to obtain DNA for genotyping. To maximise survival, eggs were allowed to develop into pupae at low larval density [51] at which time they were sacrificed for genotype analysis. One cross was excluded from the analysis because one of the males failed to achieve 3 copulations within 2 hours. A further 7 crosses were excluded from the analysis because there was allele sharing between the female and one (or both) males which precluded assignment of offspring paternity. This left 22 families for which we could estimate $P_{2}$.

\section{Microsatellite genotyping}

DNA isolation from male middle tibia, female abdomens, and whole pupae was conducted with slight modification to the protocol of Holehouse et al. [53]. Briefly, the tissue was ground in 45 of $\mu \mathrm{l}$ Tris-EDTA buffer (pH 8.0) with 10 $\mu \mathrm{l}$ of $20 \mathrm{mg} / \mathrm{ml}$ proteinase $\mathrm{K}$ (Sigma Aldrich) and then incubated for 30 minutes at $55^{\circ} \mathrm{C}$ and 10 minutes at $100^{\circ} \mathrm{C}$. Multiplex PCR was performed according to 
Wright et al. [52] using a 1:10 dilution of extracted DNA and $10 \mu \mathrm{M}$ primers for ms-039, ms-090, ms-301A, and ms-402 (Table 1). PCR conditions were as follows: denaturation at $94^{\circ} \mathrm{C}$ for 2 minutes followed by 30 cycles of $94^{\circ} \mathrm{C}$ for $30 \mathrm{sec}, 55^{\circ} \mathrm{C}$ for $30 \mathrm{sec}, 72^{\circ} \mathrm{C}$ for $65 \mathrm{sec}$, followed by $72^{\circ} \mathrm{C}$ for 10 minutes. Fluorescently labeled PCR products were separated on a 3100 DNA Analyzer (Applied Biosystems) and analyzed with Genescan 3.1.2 software (Applied Biosystems). Alleles from all 4 loci were scored for all individuals in the study.

\section{Statistical analysis}

We tested for consistent differences in male size between first and second males in the mating trials using paired $t$ tests for absolute eyespan and thorax length. Second male sperm precedence $\left(P_{2}\right)$ was calculated as the proportion of offspring from a brood sired by the second male to have mated with the female $\left(P_{2}=n_{2} /\left(n_{1}+n_{2}\right)\right.$, where $n_{i}$ equals the number of offspring from male $i$ ). We tested for heterogeneity in $P_{2}$ among families using the $G_{H}$ statistic from a replicated goodness-of-fit test, compared against a $\chi^{2}$ distribution with number of families minus 1 degrees of freedom ([54], p. 715). The numbers of offspring sired by each male, rather than proportions, were used in the derivation of $G_{H}$. Each family was also tested for its adherence to a null-hypothesis of $P_{2}=0.5$ (i.e. paternity distributed at random) using 2-tailed binomial tests ([55], p. 533). Extreme values of $P_{2}$ ( 1 or 0$)$ may have arisen from functional infertility of the first or second male, respectively. We therefore asked whether the incidence of these events were significantly greater than that expected given the standing level of functional infertility in the population for males mated 3 times (estimated as $12.5 \%$ by Baker et al. [38]). We note that this test is conservative as Baker et al. [38] defined males as infertile if their hatching success was $<10 \%$. Correlates of sperm precedence were performed using arcsine-transformed data $\left(\arcsin \sqrt{P_{2}}\right)$. Sta- tistical analyses were performed using JMP software (version 5, SAS Institute, Gary, NC, USA).

\section{Authors' contributions}

LSC conceived the study, contributed to the design and execution of the experiment, and helped to draft the manuscript. SC conducted the statistical analysis and drafted the manuscript. EM performed the experiment and carried out the molecular genetic analyses. TC, KF and AP jointly conceived the study with LSC, participated in the design and coordination, and helped to draft the manuscript. All authors have read and approved the final manuscript.

\section{Acknowledgements}

This work was funded by a BBSRC research grant to TC, KF and AP, employing LSC, SC and EM. The authors thank G Wilkinson for providing the microsatellite primer sequences and PCR conditions.

\section{References}

I. Birkhead TR, Moller AP: Sperm competition in birds: evolutionary causes and consequences Academic Press: London; 1992.

2. Simmons LW, Siva-Jothy MT: Sperm competition in insects: mechanisms and the potential for selection. In Sperm competition and sexual selection Edited by: Birkhead TR, Møller AP. San Diego, Calif: Academic Press; 1998:341-434.

3. Simmons LW: Sperm competition and its evolutionary consequences in the insects Princeton: Princeton University Press; 200I.

4. Doussard DE, Hands CA, Meinwald J, Eisner T: Pheromonal advertisement of a nuptial gift by a male moth (Utetheisa ornatrix). Proc Natl Acad Sci USA 1991, 88:9224-9227.

5. Lewis SM, Austad SN: Sources of intraspecific variation in sperm precedence in red flour beetles. Am Nat 1990, 135:35I-359.

6. Danielsson I: Antagonistic pre- and post-copulatory sexual selection on male body size in a water strider (Gerris lacustris). Proc $R$ Soc Lond B 2000, 268:77-8I.

7. Boorman E, Parker GA: Sperm (ejaculate) competition in Drosophila melanogaster, and the reproductive value of females to males in relation to female age and mating status. Ecol Entom 1976, I: 145-155.

8. Cook PA, Harvey IF, Parker GA: Predicting variation in sperm precedence. Phil Trans R Soc Lond B 1997, 352:77I-8I0.

9. Wigby S, Chapman T: Sperm competition. Curr Biol 2004, 14:100-103.

10. Wedell N: Sperm competition in butterflies and moths. In Insect Evolutionary Ecology Edited by: Fellowes M, Holloway G, Rolff J. London: CABI Publishing; 2005:49-8I.

II. Evans JP, Magurran AE: Patterns of sperm precedence and predictors of paternity in the Trinidadian guppy. Proc $R$ Soc Lond B 200I, 268:719-724.

Table I: Microsatellite loci used in this study; repeat motif, allele size range in base pairs, and nucleotide sequence all from Wright et al. [52].

\begin{tabular}{|c|c|c|c|}
\hline Locus & Repeat Motif & Product Size (bp) & Primer Sequence \\
\hline \multirow[t]{2}{*}{ ms-039 } & {$[\mathrm{AC}] 2 \mathrm{AA}[\mathrm{AC}] 4 \mathrm{GCW}[\mathrm{CA}] 3 \mathrm{~A}[\mathrm{AC}] 7 \mathrm{AT}[\mathrm{AC}] \mathrm{ITC}[\mathrm{AC}] 2$} & 147 & F:FAM-AATCACAACGCTAACGAGTCA \\
\hline & & & R:ATGCTTCAACGCTTACCTACC \\
\hline \multirow[t]{2}{*}{$\mathrm{ms}-090$} & [GT]।IGA[GT]3GG[GT]4AT[GT]। & 197 & F:FAM-TCTTGCCTTTGCCACACTAA \\
\hline & & & R:TGGGAAATGTGAGTTTACTTAAACAGT \\
\hline \multirow[t]{2}{*}{$\mathrm{ms}-30 \mathrm{IA}$} & {$[\mathrm{AC}] 8 \mathrm{AT}[\mathrm{AC}] 3 \mathrm{TC}[\mathrm{AC}] 2$} & 138 & F:HEX-TTCAGCACTAAATGCAGCAGA \\
\hline & & & R:GCACTTAACATGCGATGAGG \\
\hline \multirow[t]{2}{*}{$m s-402 A$} & I. $[\mathrm{CAA}] 2 \mathrm{ATA}[\mathrm{CAA}] 8 \mathrm{CAG}[\mathrm{CAA}] 2$ & 205 & F:HEX-CCAAATGGGCCACATTATTC \\
\hline & 2. $[A C] 8$ & & R:AGGAAAGTGGATGCATTCGT \\
\hline
\end{tabular}


12. Wedell N, Gage MJG, Parker GA: Sperm competition, male prudence and sperm-limited females. Trends Ecol Evol 2002, 17:313-320.

13. Dewsbury DA: Ejaculate cost and male choice. Am Nat 1982, I 19:60I-6I0.

14. Mesterton-Gibbons M: On sperm competition games: incomplete fertilization risk and the equity paradox. Proc $R$ Soc Lond B 1999, 266:269-274.

15. Levitan DR, Petersen C: Sperm limitation in the sea. Trends Ecol Evol 1995, | 0:228-23|.

16. Yund PO: How severe is sperm limitation in natural populations of marine free-spawners? Trends Ecol Evol 2000, 15:10-13.

17. Levitan DR: Density-dependent sexual selection in external fertilizers: variances in male and female fertilization success along a continuum from sperm limitation to sexual conflict in the Sea urchin Strongylocentrotus franciscanus. Am Nat 2004, 164:298-309.

18. García-González F: Infertile matings and sperm competition: the effect of "nonsperm representation": on intraspecific variation in sperm precedence patterns. Am Nat 2004, 164:457-472.

19. Wilkinson GS, Dodson GN: Function and evolution of antlers and eye stalks in flies. In The Evolution of Mating Systems in Insects and Arachnids Edited by: Choe J, Crespi B. Cambridge: Cambridge University Press; 1997:310-328

20. Wilkinson GS: Genetic consequences of sexual selection in stalk-eyed flies. In Model systems in behavioural ecology. Integrating conceptual, theoretical, and empirical approaches Edited by: Dugatkin LA. Princeton: Princeton University Press; 2001:72-91.

21. Chapman T, Pomiankowski A, Fowler K: Stalk-eyed flies. Curr Biol 2005, I 5:533-535.

22. Cotton S, Pomiankowski A: Do insect sexual ornaments demonstrate heightened condition dependence? In Insect Evolutionary Ecology Edited by: Fellowes M, Holloway G, Rolff J. London: CABI Publishing; 2005:3।-47.

23. Baker RH, Wilkinson GS, DeSalle R: The phylogenetic utility of different types of molecular data used to infer evolutionary relationships among stalk-eyed flies (Diopsidae). Syst Biol 200I, 50:87-105.

24. Burkhardt $D$, de la Motte I: Big 'antlers' are favoured: female choice in stalk- eyed flies (Diptera, Insecta), field collected harems and laboratory experiments. J Comp Physiol A 1988 162:649-652.

25. Wilkinson GS, Reillo PR: Female preference response to artificial selection on an exaggerated male trait in a stalk-eyed fly. Proc R Soc Lond B 1994, 255: I-6.

26. Wilkinson GS, Kahler $\mathrm{H}$, Baker RH: Evolution of female mating preferences in stalk-eyed flies. Behav Ecol 1998, 9:525-533.

27. Hingle A, Fowler K, Pomiankowski A: Size-dependent mate preference in the stalk-eyed fly Cyrtodiopsis dalmanni. Anim Behav 200I, 6I:589-595.

28. Hingle A, Fowler K, Pomiankowski A: The effect of transient food stress on female mate preference in the stalk-eyed fly Cyrtodiopsis dalmanni. Proc $R$ Soc Lond B 200I, 268: 1239-I244.

29. Cotton S, Rogers DW, Small J, Pomiankowski A, Fowler K: Variation in preference for a male ornament is positively associated with female eyespan in the stalk-eyed fly Diasemopsis meigenii. Proc $R$ Soc Lond B 2006, 273:1287-I292.

30. Burkhardt $D$, de la Motte I: How stalk-eyed flies eye stalk-eyed flies: observations and measurements of the eyes of Cyrtodiopsis whitei (Diopsidae, Diptera). J Comp Physiol A 1983, | 5 |:407-42 I.

31. Panhuis TM, Wilkinson GS: Exaggerated eyespan influences male contest outcome in stalk-eyed flies. Behav Ecol Sociobiol 1999, 46:22I-227.

32. Meier R, Baker RH: A cladistic analysis of Diopsidae (Diptera) based on morphological and DNA sequence data. Insect Syst Evol 2002, 33:325-336.

33. Burkhardt $D$, de la Motte I: Selective pressures, variability, and sexual dimorphism in stalk-eyed flies (Diopsidae). Naturwissenschaften 1985, 72:204-206.

34. Grant CA: The evolution of multiple mating in the stalk-eyed fly, Cyrtodiopsis dalmanni. In Ph.D. thesis University College London, Department of Biology; 2003.
35. Reguera P, Pomiankowski A, Fowler K, Chapman T: Low cost of reproduction in female stalk-eyed flies, Cyrtodiopsis dalmanni. J Ins Physiol 2003, 50:103-108.

36. Rogers DW, Grant CA, Chapman T, Pomiankowski A, Fowler K: The influence of male and female eyespan on fertility in the stalkeyed fly Cyrtodiopsis dalmanni. Anim Behav 2006 in press.

37. Kotrba M: Sperm transfer by spermatophore in the Diptera: new results from the Diopsidae. Zool J Linn Soc 1996, I 1 7:305-323.

38. Baker RH, Ashwell RIS, Richards TA, Fowler K, Chapman T, Pomiankowski $A$ : Effects of multiple mating and male eye span on female reproductive output in the stalk-eyed fly Cyrtodiopsis dalmanni. Behav Ecol 200I, I 2:732-739.

39. Lorch PD, Wilkinson GS, Reillo PR: Copulation duration and sperm precedence in the stalk-eyed fly Cyrtodiopsis whitei (Diptera: Diopsidae). Behav Ecol Sociobiol 1993, 32:303-3II.

40. Wilkinson GS, Fry CL: Meiotic drive alters sperm competitive ability in stalk- eyed flies. Proc R Soc Lond B 200I, 268:2559-2564.

4I. Rogers DW, Chapman T, Fowler K, Pomiankowski A: Matinginduced reduction in accessory reproductive organ size in the stalk-eyed fly Cyrtodiopsis dalmanni. BMC Evol Biol 2005, 5:37.

42. Harvey IF, Parker GA: 'Sloppy' sperm mixing and intraspecific variation in sperm precedence $\left(\boldsymbol{P}_{2}\right)$ patterns. Proc $R$ Soc Lond $B$ 2000, 267:2537-2542.

43. Eberhard WG: Sexual selection and animal genitalia Cambridge MA; Harvard University Press; 1985.

44. Otronen M: Sperm numbers, their storage and usage in the fly Dryomyza anilis. Proc R Soc Lond B 1997, 264:777-782.

45. Danielsson I, Askenmo C: Male genital traits and mating interval affect male fertilization success in the water strider Gerris lacustris. Behav Ecol Sociobiol 1999, 46:149-I56.

46. Baker RH, Denniff M, Futerman P, Fowler K, Pomiankowski A, Chapman $\mathrm{T}$ : Accessory glands influence time to sexual maturity and mating frequency in the stalk-eyed fly, Cyrtodiopsis dalmanni. Behav Ecol 2003, 14:607-6II.

47. Rogers DW, Baker RH, Chapman T, Denniff M, Pomiankowski A Fowler K: Direct and correlated responses to artificial selection on male mating frequency in the stalk-eyed fly Cyrtodiopsis dalmanni. J Evol Biol 2005, 18:642-650.

48. Fry CL: Juvenile hormone mediates a trade-off between primary and secondary sexual traits in stalk-eyed flies. Evol Dev 2006, 8: $|9|-20 \mid$.

49. David P, Bjorksten T, Fowler K, Pomiankowski A: Conditiondependent signalling of genetic variation in stalk-eyed flies. Nature 2000, 406:186-188.

50. Presgraves DC, Severance E, Wilkinson GS: Sex chromosome meiotic drive in stalk-eyed flies. Genetics 1997, | 47: | |69- | I80.

5I. Cotton S, Fowler K, Pomiankowski A: Condition dependence of sexual ornament size and variation in the stalk-eyed fly Cyrtodiopsis dalmanni (Diptera: Diopsidae). Evolution 2004, 58:1038-1046.

52. Wright TF, Johns PM, Walters JR, Lerner AP, Swallow JG, Wilkinson GS: Microsatellite variation among divergent populations of stalk-eyed flies, genus Cyrtodiopsis. Genet Res 2004, 84:27-40.

53. Holehouse KA, Hammond RL, Bourke AFG: Non-lethal sampling of DNA from bumble bees for conservation genetics. Insect Soc 2003, 50:277-285.

54. Sokal RR, Rohlf FJ: Biometry 3rd edition. New York: Freeman; 1995.

55. Zar JH: Biostatistical analysis 3rd edition. London: Prentice-Hall; 1996. 